\title{
Persona's Dilemma between Academics and practitioners - Ideation Guidelines for Co-designing Future Technologies Workshops
}

\author{
Parisa Saadati \\ University of West London \\ London W5 5RF \\ parisa.saadati@uwl.ac.uk
}

\author{
Jose Abdelnour-Nocera \\ University of West London \\ London W5 5RF \\ Jose.Abdelnour-Nocera@uwl.ac.uk
}

\author{
Torkil Clemmensen \\ Copenhagen Business School \\ Denmark \\ tc.digi@cbs.dk
}

\begin{abstract}
The introduction of Industry 4.0 interactive technologies and automated systems in complex organizations have imposed novel challenges and burdens on academics and industrial practitioners for developing systems that work for future workplaces. Developing such systems need sufficient knowledge and understanding of the trends and technological developments and viability from industry and academic experts before introducing them to the general population. Co-designing workshops with employees and users supported by various design tools can provide better ideation for designing future scenarios. We conducted a qualitative study to analyze academics' and industrial practitioners' views on a persona as a design tool during a conference workshop. These participants empirically test the co-creation of personas and find conceptual differences between the groups in their tool use. We propose guidelines on using personas for idea management in the co-design of future scenarios using pre and post-workshop surveys and workshop transcripts to code and clustered our findings. The conclusion is that considering the differences in academics' and industrial practitioners' perspectives, using the right design tools for ideation in a prepared environment for a combined team is substantial and can lead to designing positive experiences in future workplaces.
\end{abstract}

Future technologies, Personas, Co-designing workshops, Academics, Practitioners.

\section{INTRODUCTION}

Advanced machines can simultaneously perform multiple functions - a benefit that human beings cannot provide (Parasuraman et al., 2000). Industry 4.0 transition will bring more autonomous future technologies. Despite the numerous benefits of automation, it is essential to determine which functions should be automated and to what extent (Parasuraman et al., 2000). Therefore, there is a burden on interactive system designers for sound design work for its users. The analysis presented in this article has been developed to elicit different perspectives from academics and industry practitioners in understanding the nature and influence of using personas as a design tool in professional design work for future technologies. In this study, academics and industrial practitioners refer to individuals who engage in design activities at any level: the former with both academic and problem-solving motivations and the latter with an interest or responsibilities to find solutions to domain-specific problems.

In comparison, academics create more insights on selecting and evaluating design ideas as fundamental skills. In practice, the success of industry practitioners is based on experience rather than theoretical knowledge (Inie and Dalsgaard, 2020). However, despite disputes and differences between stakeholders' internal cultures or communication (Sarin and O'Connor, 2009), a common understanding and a shared vision to develop a new design must be facilitated to an efficient outcome.

\subsection{Future Technologies}

Predicting how the industries will develop or what future services will look like should be based on grounded foundations. The result can be sufficient knowledge about future workplace interactive technologies to recognize desirable or undesirable possibilities. The decision-makers can use this result as a broad understanding to avoid the negative consequences of using such future technologies (Jenkins et al., 2020).

In the design process, it is popular to use simple story studies (Jenkins et al., 2020; Kymalainen et al., 2016) based on trends and events to capture future possibilities. This can generate ideas by facilitating brainstorming sessions for more realistic scenarios or adopting 'blue sky thinking' to turn current trends and signals into the future experience (Jenkins et al., 2020). The result most probably impacts people's decision-making and increases creative capacity when considering the future.

\subsection{Co-designing and selecting the right tool.}

Co-design is used as a central approach in designing services in support of multi-disciplinary collaboration or future technologies. It may be defined as the creativity of designers and people 
not trained in design working together in the design development process' (Sanders and Stappers, 2008). Co-designing artifacts with design tools is becoming increasingly popular for generating and managing ideas to establish a shared understanding and shared vision and goals in the early stages of an innovation process (Rygh and Clatworthy, 2019). However, using these approaches through these design tools to design new technologies is challenging and needs a facilitated environment to support the participant to ensure success.

Using tangible design tools can establish a shared 'language' through physical form when verbal communication fails due to professional terminology and misalignments between different professional working cultures (Jenkins et al., 2020; Kymalainen et al., 2016; Rygh and Clatworthy, 2019). On the one hand, it is vital to match the design tools and the people interacting with them in the co-designing sessions. Using these design tools is often developed on a trial-and-error basis, resulting in low engagement, trust, and interaction with the participating stakeholders (Rygh and Clatworthy, 2019). An example of such tools is the persona, a ubiquitous design tool in many studies and, not surprisingly, a widespread tangible tool in the industry. (Jansen et al., 2020; Nielsen, 2019; Tomlin, 2018)

A persona is a communicational tool typically used within User-Centred Design (Nielsen, 2019), which was introduced by Alan Cooper (Cooper, 2004) for the first time for mass-market software development. Personas in co-design projects begun to include users and others in either persona inceptions or assemblies or its deployment. Personas could provide a deeper understanding of improving the quality of work and the work environment for future scenarios (Cajander et al., 2015).

This paper asks the research question: What are the main differences in academics' and industrial practitioners' points of view on common design tools for ideation? What are the main requirements for preparing the environment for co-creation of personas and find conceptual differences between the groups in their tool use?

\section{METHODOLOGY}

We investigated a selection of design tools with particular attention to personas for co-designing (Simonsen, Jesper and Robertson, Toni, 2012) workshops for future scenarios. The selection criteria for these tools are based on suitability with the work domains of the designer, namely Industry 4.0, and automation, and convenience for the type, space, and duration of the workshops. We conducted a 5-hour workshop during a well-known $\mathrm{HCl}$ conference on co-designing personas for user experience (UX) and engagement in automation to gather this input. We also invited industry practitioners to join us to get their insights on the topic at hand. Due to COVID-19 outbreak, this workshop was run on an online platform virtually. The goals of the workshop, participants discussed:

- Presenting different views on using idea management workshops as a fundamental practice for future scenarios for industry practitioners and academics.

- Offering guidelines on using Personas as a design tool for idea management in the co-design of future scenarios.

\subsection{Participant and Data collection process}

The participants in this workshop were three industry practitioners from different companies, one doctoral student, three active researchers, and one post-doctorate researcher. The industry practitioners were a consultant for government projects, an innovation consultant with experience in innovation and user experience for a busy airport, and a business developer representing the UK-based start-up working with the high-ranking companies for the industry 4.0 transition.

We employed the Nominal Group Technique (Delbecq et al., 1975) for data collection during the workshop. This method is a structured method for group brainstorming that encourages contribution from everyone and quicker decision-making on the important relative issue, problem, or solutions. This technique helped all participants to have their contributions first and then discuss them with others. We can divide the workshop activities into three phases.

(i) Introduction of the participants and the workshop goals. Most participants presented their findings of the topic as part of their contribution, while others could ask questions and discuss their opinion on utilizing personas in the design process!

(ii) Idea generation phase. In this phase, we used a personas template (Nielsen, 2019) and invited the participants to co-design two personas for a defined scenario. They were discussions lead to questions on the situation, obstacles, domain context, users, and even the technology.

(iii) Idea sharing phase. Each participant described their notes on the scenario and raised the issues. The template allowed the participant to cluster their ideas under each relevant heading. 
After the activity, the facilitator sent a survey asking participants for insights at the end of the workshop. We clustered similar insights/findings under the initial categories.

\section{WORKSHOP RESULT}

The workshop's main activity focused on codesigning personas for a scenario introduced by the UK-based start-up focused on retail shopfloor robots in future supermarkets. During the Idea Generation phase, the workshop participants individually reported 50 different insights based on their previous works or research about using personas as a design tool. Subsequently, during the Idea Sharing phase, the participants discussed their different views on each insight. This number decreased to 21 as the number of duplicated insights was removed from the list. In this phase, participants benefited from each other's point of view and agreed on the number of the points raised as disagreement before. After this discussion, the final main categories were clustered into 9 insights (covered in Tables 1 and 2). Table 1 shows the main disagreement and table 2 is a finalized summary of the agreed insights on standard practices.

Table 1. Disagreed insights between the academics and industry practitioners on the designing personas.

\begin{tabular}{|c|c|}
\hline Academics & Industry practitioners \\
\hline \multicolumn{2}{|c|}{ Data driven persona vs proto personas } \\
\hline $\begin{array}{l}\text { Incorrect practices on } \\
\text { personas by industry. } 3 \\
\text { votes }\end{array}$ & $\begin{array}{l}\text { Proto personas and } \\
\text { reverse engineering as a } \\
\text { common practice. } 4 \text { votes }\end{array}$ \\
\hline \multicolumn{2}{|c|}{ Helpfulness of personas in designing future scenarios } \\
\hline $\begin{array}{l}\text { Co-designing for future } \\
\text { scenarios better than the } \\
\text { current ones. } 6 \text { votes }\end{array}$ & $\begin{array}{lrr}\text { Not easy to } & \text { create } \\
\text { personas for } & \text { new } \\
\text { segments. } 2 \text { votes } & \\
\end{array}$ \\
\hline \multicolumn{2}{|c|}{ The number of personas to design } \\
\hline $\begin{array}{l}\text { Businesses should } \\
\text { prioritize the number of } \\
\text { personas. } 3 \text { votes }\end{array}$ & $\begin{array}{l}\text { No particular number to } \\
\text { follow; the team will } \\
\text { decide on this number. } 4 \\
\text { votes }\end{array}$ \\
\hline \multicolumn{2}{|c|}{ Type of data collection for personas } \\
\hline 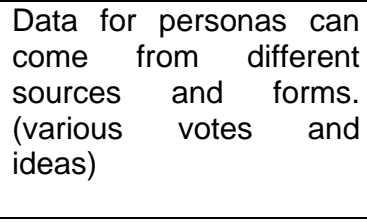 & $\begin{array}{l}\text { Using segmentation in } \\
\text { busy domains is } \\
\text { impossible, but alternative } \\
\text { sources are available. } \\
\text { (agreement on some of } \\
\text { the alternative sources) }\end{array}$ \\
\hline
\end{tabular}

Table 2. Agreed insights between the academics and industry practitioners on co-designing personas.

\begin{tabular}{|l|}
\hline \multicolumn{1}{|c|}{ Insights agreed } \\
\hline Personas can be used in iterative design and for \\
testing the systems. \\
\hline $\begin{array}{l}\text { A.I. personas should consider future scenarios, } \\
\text { specifically for technologies that have livelihood about }\end{array}$ \\
\hline
\end{tabular}

their jobs and work alongside the human worker (e.g., Robots, A.I. engines).

Photo personas can transfer pre-conceptions about the users to the developers, or it can touch cultural sensitivity.

Data collection is not always accessible, but having a context, situation, obstacles, and a scenario is essential for co-designing personas.

A shared, engaging environment that people can design together is important for co-designing the personas and can increase the chance of practicable personas.

\section{GUIDELINES ON USING PERSONAS FOR IDEATION FOR FUTURE SCENARIOS CO- DESIGNING WORKSHOPS.}

We noticed that the ideation process could be sped up by providing a context, scenario, and better facilitation during the codesigning workshop. Previous studies cover how this process can be more comfortable and quicker for the participants (Inie and Dalsgaard, 2020; Rygh and Clatworthy, 2019). We add to this literature by presenting insights from our workshop.

Selecting the right design tool: Selecting design tools for co-design for future scenarios should be based on the workshop medium (i.e., online or face-to-face) and participants' familiarity with the design process and tools (i.e., professional, expert, typical end-users). Participants select the tools they are more confident with and tools they may not need specific knowledge or literacy for working with (e.g., sticky notes). Adding more tangible elements (Rygh and Clatworthy, 2019) such as prestructured cards and easy-to-use collaborative tools can always be beneficial.

\section{Preparing the environment to ideate:}

Whether it is a face-to-face or virtual codesign workshop, participants should understand the scenario, products, and future system's domain and environment. For example, using employees as participants facilitate familiarity with the work domain. However, preparing all participants with brainstorming, group discussion, etc can provide

them with the exemplary scenario and situation to generate and manage their ideas. Nevertheless, during our workshop, we have noticed that we do not only need collaborative design tools, but we also need facilitation with a communication system, ideally recording the sessions and discussions.

\section{Refining the tools for future scenarios:}

We used a persona template (Nielsen, 2019), which has a part for the scenario, unlike many common 
persona templates widely available. In particular, this section was helpful in a virtual co-design workshop as it provides a platform to test the persona in the given scenario. Service design guides introduce more useful design tools than personas with more actors to look at different levels in the user journey (i.e., user stories and blueprint), thus having a new investigation on designing more contextual personas with the new requirements is needed (Cajander et al., 2015). We used an approach towards a scenario and a persona which brought more interaction into the workshop. Consequently, it brought up more issues to deal with (e.g., trust, hierarchies, and persona's limitations) rather than using only one of them. We agree that adding the two sections for the context data and external and internal sources to build the personas is necessary, considering more online collaborations to come.

\section{$\underline{\text { Personas for A.I.: }}$}

Industry practitioners supported the idea of creating 'Personas for A.I.' in the introduction phase, which was challenged by some academics at the beginning of the workshop. Practitioners emphasized the importance of A.I. behavior in different domains. For example, in airports, A.I. systems are associated with a level of risk as they will take away some controls from human counterparts. Therefore, there is a need for generating a persona for such systems, one of the participants said. Likewise, UK-based startup's operation team has created different robot profiles in the airport and retail's environment based on A.I. behaviour. Ultimately, one of the consultants suggested looking into a new design and phrase for personas for technology to be called 'Techsona' (Technology Persona).

\section{Instruction for facilitator during the activities:}

The facilitator's role is significant in such co-design workshops to help and manage the ideation process. Knowing the main topics to cover or being open about the new topic can create more real personas, such as raising privacy or GDPR topics while co-designing personas for the retail shopfloor robot's scenario in this workshop. As agreed by participants, facilitators should be carefully selected and trained to run the co-design sessions. They need to follow instructions and use carefully selected activities. A participant who played the facilitator role in the workshop, after the co-design activity, suggested that facilitators should supervise the outcome and tidy up the outcome of the idea management activity. We also suggest collaborations between academics and industry for co-designing future technologies. For instance, the facilitator can be selected from academia working with the industry. To make the personas or any other design tools in line with the organization's UX, providing a short, easy-tounderstand template should be considered. One of the academics in the workshop believed that the UX\AI goals could help shape the use-cases if facilitated with a proper exercise.

\section{Importance of scenarios:}

In line with the environment preparation and facilitator's instructions, it is essential to plan for a scenario for every co-design workshop for future scenarios. This can help the participant access context and a road map to look forward and share more relevant information. One academic believed personas should be tested on scenarios and refined again and again for an optimal outcome closer to reality for future technologies. The industry practitioners in the team supported his idea strongly.

\section{Data gathering for persona designing:}

There is a need to use data-driven design tools to co-design for future scenarios. Accessibility to this data in the templates, collaborative platforms, or any other forms can provide better context and understanding for the participants before using these tools.

\section{CONCLUSION}

We explored empirical differences in academics' and industry practitioners' perspectives in using persona as a design tool to ideate future scenarios. We analyzed the collected data from a conference workshop. Using participant's point of views, we recommend a guideline for running co-design workshops. Our analysis indicated that while these two groups may, on the behavioural level, use common design tools in the same fashion, there are important differences between them in the thinking, ideation, prototyping, and overall design process. We can argue that (1) there is a need to involve both academics and industry practitioners in co-design ideation workshops for emergent future systems, (2) design tools used should be linked explicitly to specific contexts, scenarios, or situations to provide decision information relevant to the specific domain and environment, (3) using data-driven design tools based on the current trends and events may facilitate consensus about a design reality. Also, the facilitators of co-design ideation workshops have a critical role in leading the various designers and the overall session to an optimal outcome. 


\section{REFERENCES}

Cajander, A., Larusdottir, M., Eriksson, E., Nauwerck, G., 2015. Contextual Personas as a Method for Understanding Digital Work Environments, in: Abdelnour Nocera, J., Barricelli, B.R., Lopes, A., Campos, P., Clemmensen, T. (Eds.), Human Work Interaction Design. Work Analysis and Interaction Design Methods for Pervasive and Smart Workplaces, IFIP Advances in Information and Communication Technology. Springer International Publishing, Cham, pp. 141-152. https://doi.org/10.1007/978-3-319-27048-7 10

Cooper, A., 2004. The Inmates are Running the Asylum: Why High-tech Products Drive Us Crazy and How to Restore the Sanity., 1st Edition. ed. Sams.

Delbecq, A.L.., Van De Ven, A.H., Gustafson, D.H., 1975. Group techniques for program planning: a guide to nominal group and delphi processes, Management applications series. Scott, Foresman.

Inie, N., Dalsgaard, P., 2020. How Interaction Designers Use Tools to Manage Ideas. ACM Trans. Comput.-Hum. Interact. 27, 7:1-7:26. https://doi.org/10.1145/3365104

Jansen, B.J., Salminen, J.O., Jung, S.-G., 2020. Data-Driven Personas for Enhanced User Understanding: Combining Empathy with Rationality for Better Insights to Analytics. Data and Information Management 4, 1-17. https://doi.org/10.2478/dim-2020-0005

Jenkins, T., Boer, L., Brigitta Busboom, J., Simonsen, I. \&Oslash;stby, 2020. The Future Supermarket: A Case Study of Ethnographic Experiential Futures, in: Proceedings of the 11th Nordic Conference on Human-Computer Interaction: Shaping Experiences, Shaping Society. Presented at the NordiCHI '20: Shaping Experiences, Shaping Society, ACM, Tallinn Estonia, pp. 1-13. https://doi.org/10.1145/3419249.3420130

Kymalainen, T., Kaasinen, E., Aikala, M., Hakulinen, J., Heimonen, T., Paunonen, H., Ruotsalainen, J., Lehtikunnas, L., Mannonen, P., 2016. Evaluating Future Automation Work in Process Plants with an Experience-Driven Science Fiction Prototype, in: 2016 12th International Conference on Intelligent Environments (I.E.). Presented at the 2016 12th International Conference on Intelligent Environments (I.E.), IEEE, London, United Kingdom, pp. 54-61. https://doi.org/10.1109/IE.2016.17
Nielsen, L., 2019. Personas - User Focused Design, Human-Computer Interaction Series. Springer London, London. https://doi.org/10.1007/978-1-4471-7427-1

Rygh, K., Clatworthy, S., 2019. The Use of Tangible Tools as a Means to Support Codesign During Service Design Innovation Projects in Healthcare, in: Pfannstiel, M.A., Rasche, C. (Eds.), Service Design and Service Thinking in Healthcare and Hospital Management. Springer International Publishing, Cham, pp. 93-115. https://doi.org/10.1007/9783-030-00749-2 7

Sanders, E.B.-N., Stappers, P.J., 2008. Co-creation and the new landscapes of design. CoDesign 4, 5-18.

https://doi.org/10.1080/15710880701875068

Sarin, S., O'Connor, G.C., 2009. First among Equals: The Effect of Team Leader Characteristics on the Internal Dynamics of Cross-Functional Product Development Teams. Journal of Product Innovation Management 26, 188-205. https://doi.org/10.1111/j.15405885.2009.00345.x

Simonsen, Jesper and Robertson, Toni, 2012. Routledge international handbook of participatory design. Routledge.

Tan, W., Boy, G.A., 2018. Tablet-Based Information System for Commercial Aircraft: Onboard Context-Sensitive Information System (OCSIS), in: Harris, D. (Ed.), Engineering Psychology and Cognitive Ergonomics, Lecture Notes in Computer Science. Springer International Publishing, Cham, pp. 701-712. https://doi.org/10.1007/978-3-319-91122-9_55 